\title{
Effects of Variable Viscosity on Hydromagnetic Boundary Layer along a Continuously Moving Vertical Plate in the Presence of Radiation and Chemical Reaction
}

\author{
Utpal Jyoti Das
}

Department of Mathematics, Rajiv Gandhi University, Arunachal Pradesh, India.

Email: utpaljyotidas@yahoo.co.in

Received November $6^{\text {th }}, 2012$; revised December $7^{\text {th }}$, 2012; revised December $18^{\text {th }}, 2012$

\begin{abstract}
The flow and heat transfer of an incompressible viscous electrically conducting fluid over a continuously moving vertical infinite plate with uniform suction and heat flux in porous medium, taking account of the effects of the variable viscosity, has been considered. The solutions are obtained for velocity, temperature, concentration and skin friction. It is found that the velocity increases as the viscosity of air or porous parameter increases whereas velocity decreases when Schmidt number increases. The skin friction coefficient is computed and discussed for various values of the parameters.
\end{abstract}

Keywords: MHD; Variable Viscosity; Radiation; Porous Medium; Schmidt Number

\section{Introduction}

The problem of heat and mass transfer in the boundary layer induced by a moving surface in a quiescent fluid is important in many engineering applications. For examples, in the extrusion of polymer sheet from a dye, the cooling of an infinite metallic plate in a cooling path, glass blowing continuous casting and spinning of fibers. Sakiadis [1] studied the boundary layer flow over a continuous solid surface moving with constant velocity in an ambient fluid. The flow is quite different from the boundary layer flow over a semi-infinite flat plate due to the entrainment of the ambient fluid. Tsou et al. [2] presented a combined analytical and experimental study of the flow and temperature fields in the boundary layer on a continuous moving surface. Erickson et al. [3] extended Sakiadis problem to include blowing or suction at the moving surface. Crane [4] studied the boundary layer flow caused by a stretching sheet whose velocity varies linearly with the distance from a fixed point on the surface. The magnetohydrodynamics of an electrically conducting fluid is encountered in many problems in geophysics, astrophysics, engineering applications and other industrial areas. Engineers employ magnetohydrodynamics principles in the design of heat exchangers, pumps, in space vehicle propulsion, thermal protection, control and re-entry and in creating novel power generating systems. In many metallurgical processes involve the cooling of many continuous strips or filaments by drawing them through an electrically conducting fluid subject to a magnetic field, the rate of cooling can be controlled and final product of desired characteristics can be achieved. Another important application of hydromagnetics to metallurgy lies in the purification of molten metals from non-metallic inclusions by the application of a magnetic field. Kumar et al. [5] studied hydromagnetic flow and heat transfer on a continuously moving vertical plate. Sharma and Mathur [6] investigated steady laminar free convection flow of an electrically conducting fluid along a porous hot vertical infinite plate in the presence of heat source or sink. On the other hand, at high temperature the effects of radiation in space technology, solar power technology, space vehicle re-entry, nuclear engineering applications are very significant. Many processes in industrial areas occur at high temperature and the knowledge of radiation heat transfer in the system can perhaps lead to a desired product with a desired characteristic. Raptis and Massalas [7] studied the radiation effect on the unsteady magnetohydrodynamic flow of an electrically conducting viscous fluid past a plate. Chamkha [8] investigated thermal radiation and buoyancy effects on hydromagnetic flow over an accelerating permeable surface with heat source or sink. Raptis et al. [9] discussed the effect of thermal radiation on MHD asymmetric flow of an electrically conducting fluid past a semi-infinite plate. All the above studies were confined to a fluid with constant viscosity. However, it is known that this physical property may change significantly with temperature. Hossain and Munir [10] analyzed a two-di- 
mensional mixed convection flow of a viscous incomepressible fluid of temperature dependent viscosity past a vertical plate. Fang [11] studied the influence of fluid property variation on the boundary layers of a stretching surface. Hossain et al. [12] discussed the effect of radiation on free convection flow of a fluid with variable viscosity from a porous vertical plate. Mahmoud [13] studied the effects of radiation and variable viscosity on hydromagnetic boundary layer flow along a continuously moving vertical plate with suction and heat flux.

Many transport processes exist in nature and in Industrial applications in which the simultaneous heat and mass transfer occurs as a result of combined buoyancy effects of diffusion of chemical species. Chemical reaction effects on heat and mass transfer laminar boundary layer flow have been discussed by various authors [14-18] in various situations.

In this paper, the effects of variable viscosity on hydromagnetic boundary layer flow along a continuously moving vertical plate with uniform suction and heat flux has been studied.

\section{Mathematical Formulation}

Consider a steady boundary layer convective flow through porous medium of an electrically conducting visco-elastic fluid on a continuous surface, issuing from a slot and moving vertically with a uniform velocity $u_{w}$ in a fluid and heat is supplied from the plate to the fluid at a uniform rate, in the presence of a uniform magnetic field of strength $B_{0}$. Let the $x$-axis be taken along the direction of motion of the sheet and the $y$-axis be normal to the surface. The induced magnetic field is assumed to be negligible. It is assumed that there exists a first order chemical reaction between the fluid and the fluid species concentration. The physical model of the problem is shown in Figure 1.

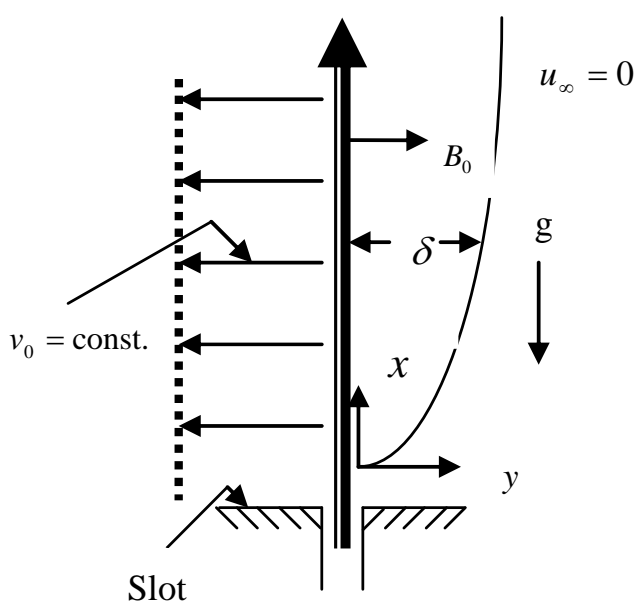

Figure 1. Physical model of the problem.
Under the above assumption, the equations for boundary layer flow are as follows:

$$
\begin{gathered}
\frac{\partial v}{\partial y}=0 \\
v \frac{\mathrm{d} u}{\mathrm{~d} y}=\frac{1}{\rho_{\infty}} \frac{\mathrm{d} u}{\mathrm{~d} y}\left(\mu \frac{\mathrm{d} u}{\mathrm{~d} y}\right)-\frac{\sigma B_{0}^{2}}{\rho_{\infty}} u \\
+g \beta\left(T-T_{\infty}\right)+g \beta_{C}\left(C-C_{\infty}\right)-\frac{\mu u}{\rho_{\infty} k^{\prime}}
\end{gathered}
$$

Equation of heat transfer:

$$
v \frac{\partial T}{\partial y}=\frac{k}{\rho C_{p}} \frac{\mathrm{d}^{2} T}{\mathrm{~d} y^{2}}-\frac{1}{\rho C_{p}} \frac{\mathrm{d} q_{r}}{\mathrm{~d} y}
$$

Equation of concentration:

$$
v \frac{\mathrm{d} C}{\mathrm{~d} y}=D \frac{\mathrm{d}^{2} C}{\mathrm{~d} y}
$$

The corresponding boundary conditions are

$$
\begin{aligned}
& u=u_{w}, v=v_{0}=\text { constant }<0, \frac{\partial T}{\partial y}=-\frac{q}{k}, \\
& C=C_{\infty}+\frac{q v_{1}}{k v_{0}} \text { at } y=0 \\
& u \rightarrow 0, T \rightarrow T_{\infty}, C=C_{\infty} \text { as } y \rightarrow \infty
\end{aligned}
$$

where $u, v$ are the velocities along $x, y$ coordinates, respectively, $g$ is the acceleration due to gravity, $\beta$ is the coefficient of thermal expansion, $\beta_{C}$ is the concentration coefficient, $T$ is the fluid temperature, $T_{\infty}$ is the temperature of the fluid far away from the plate, $\sigma$ is the electrical conductivity, $\rho_{\infty}$ is the ambient density, $\mu$ is the fluid viscosity, $q$ is the heat flux, $k$ is the thermal conductivity, $C_{p}$ is the specific heat at constant pressure, $q_{r}$ is the radiative heat transfer, $v_{0}$ is the normal velocity at the plate, $C$ is the concentration, $C_{\infty}$ is the concentration in the fluid far away from the plate, $k^{\prime}$ is the permeability of the porous medium.

From the Equation (1), we get

$$
v=-v_{0}
$$

By using Rosseland approximation, $q_{r}$ takes the form [3]

$$
q_{r}=\frac{4 \sigma^{*}}{3 k^{*}} \frac{\mathrm{d} T^{4}}{\mathrm{~d} y},
$$

where $k^{*}$ is the mean absorption coefficient and $\sigma^{*}$ is the Stefan-Boltzmann constant.

The temperature differences within the fluid assumed sufficiently small such that $T^{4}$ may be expressed as a linear function of the temperature. Expanding $T^{4}$ in Taylor series about $T_{\infty}$ and neglecting the higher order terms, we get 


$$
T^{4}=4 T_{\infty}^{3}-3 T_{\infty}^{4}
$$

By using Equations (6)-(8) then Equation (3) gives

$$
-v_{0} \frac{\mathrm{d} T}{\mathrm{~d} y}=\frac{k}{\rho_{\infty} C_{p}} \frac{\mathrm{d}^{2} T}{\mathrm{~d} y^{2}}+\frac{16 \sigma^{*} T_{\infty}^{3}}{3 \rho_{\infty} C_{p} k^{*}} \frac{\mathrm{d}^{2} T}{\mathrm{~d} y^{2}}
$$

Introducing the following non-dimensional quantities:

$$
\begin{aligned}
& \eta=\frac{\rho_{\infty} v_{0} y}{\mu_{\infty}}, f=\frac{u}{u_{w}}, \theta=\frac{T-T_{\infty}}{\left(\frac{q \mu_{\infty}}{k \rho_{\infty} v_{0}}\right)}, \phi=\frac{C-C_{\infty}}{\left(\frac{q \mu_{\infty}}{k \rho_{\infty} v_{0}}\right)}, \\
& \operatorname{Pr}=\frac{\mu_{\infty} C_{p}}{k}, G r=\frac{\mu_{\infty} g \beta \frac{q \mu_{\infty}}{\rho_{\infty} v_{w} k}}{\rho_{\infty} u_{w} v_{0}^{2}}, S c=\frac{\left(\frac{\mu_{\infty}}{\rho_{\infty}}\right)}{D}, \\
& G m=\frac{\mu_{\infty} g \beta_{c}\left(\frac{q \mu_{\infty}}{\rho_{\infty} v_{0} k}\right)}{\rho_{\infty} u_{w} v_{0}^{2}}, k_{1}=\frac{k^{\prime} v_{0}^{2}}{\left(\frac{\mu_{\infty}^{2}}{\rho_{\infty}^{2}}\right)}, R=\frac{16 a \sigma^{*} T_{\infty}^{3}}{3 k k^{*}} .
\end{aligned}
$$

In view of Equation (10), Equations (2), (9), and (4) reduce to the following non-dimensional form:

$$
\begin{gathered}
\frac{1}{\mu_{\infty}}\left(\mu f^{\prime \prime}+\mu^{\prime} f^{\prime}\right)+f^{\prime} \\
-\left(M+\frac{\mu}{\mu_{\infty} k_{1}}\right) f+G r \theta+G m \phi=0 \\
\theta=\frac{1+R}{P r} \mathrm{e}^{-\left(\frac{P r}{1+R}\right) \eta} \\
\phi=\mathrm{e}^{-S c \eta}
\end{gathered}
$$

The corresponding boundary conditions are

$$
\begin{aligned}
& \eta=0: f=1, \theta^{\prime}=-1, \varphi=1 ; \\
& \eta \rightarrow \infty: f \rightarrow 0, \theta \rightarrow 0, \varphi \rightarrow 0 .
\end{aligned}
$$

where the prime denote differentiation with respect to $\eta$ and $G r$ is the thermal Grashof number, $G m$ is the solutal Grashof number, $\mathrm{Pr}$ is the Prandtl number, $M$ is the Hartmann number, $S c$ is the Schmidt number, $k_{1}$ is the porous parameter, $R$ the radiation parameter.

The fluid viscosity $\mu(\theta)$ was assumed to obey the Reynolds model [13],

$$
\frac{\mu}{\mu_{\infty}}=\mathrm{e}^{-\alpha \theta}
$$

where $\alpha$ is a parameter depending on the nature of the fluid.

Using, Equation (13) in the Equation (11) we obtain,

$$
\left(f^{\prime \prime}-\alpha f^{\prime} \theta^{\prime}-\frac{f}{k_{1}}\right)+\mathrm{e}^{\alpha \theta}\left(f^{\prime}-M f+G r \theta+G m \phi\right)=0(16)
$$

\section{Method of Solution}

1) Case of constant viscosity:

For $\alpha=0$, from Equation (16), we have

$$
f^{\prime \prime}+f^{\prime}-\left(M+\frac{1}{k_{1}}\right) f+G r \theta+G m \phi=0
$$

Solving (17) under boundary condition (14), we get

$$
f=\left(1+r_{1}+r_{2}+r_{3}\right) \mathrm{e}^{-r_{1} \eta}-r_{2} \mathrm{e}^{-\left(\frac{P r}{1+R}\right) \eta}-r_{3} \mathrm{e}^{-S c \eta}
$$

where

$$
\begin{aligned}
& r_{1}=\frac{1+\sqrt{1+4\left(M+\frac{1}{k}\right)}}{2}, \\
& r_{2}=\frac{(1+R)^{3} \frac{G r}{P r}}{P r^{2}-P r(1+R)-\left(M+\frac{1}{k}\right)(1+R)^{2}}, \\
& r_{3}=\frac{G m}{S c^{2}-S c-\left(M+\frac{1}{k}\right)} .
\end{aligned}
$$

2) Variable viscosity case:

On taking into account the solution for temperature and concentration, we solved numerically the Equation (16) under the boundary conditions (14) using the RungeKutta fourth order technique with guessing $f^{\prime}(0)$ by shooting technique.

The skin friction coefficient is defined as

$$
C_{f}=\frac{2\left(\mu \frac{\partial u}{\partial y}\right)_{y=0}}{\rho_{\infty} v_{0} u_{w}}=2 \mathrm{e}^{-\alpha \theta(0)} f^{\prime}(0)
$$

\section{Results and Discussion}

In order to see the physical impact of the variable viscosity $(\alpha)$ on the velocity field, the graphical representation of results is important. For the purpose of discussing the effect of variable viscosity on the flow profiles within the boundary layer, numerical calculations have been carried out for various values involved in the problem with fixed values of $\mathrm{Pr}$. The value of $\mathrm{Pr}$ is taken to be 0.733 for air. The effect of $\alpha$ on dimensionless velocity $f$ are illustrated in Figures 2-4 with $G r=5, R=0.2, M=2, G m=1$.

It is observed from the Figure 2 that velocity $f$ increases as the viscosity $(\alpha)$ of air decreases. The velocity distribution attains a distinctive maximum value in the vicinity of the plate and then decreases to approach a free stream value. 
Figure 3 shows the effects of Schmidt number on the velocity profile. As the Schmidt number increases there is reduction in the fluid velocity. The fluid velocity increased and reached its maximum value at very short distance from the plate and then decreases to approach a free stream value.

The effect of the porous parameter on velocity profile is shown in Figure 4. From the figure it is observed that fluid velocity increases with the increase in the porous parameter. Table 1 present the variation of the skin friction $f^{\prime}(0)$ for various values of $\alpha$, Hartmann number $M$, radiation parameter $R$, thermal Grashof number $G r$, solutal Grashof number $G m$, Schmidt number $S c$, porous parameter $k_{1}$ with $\operatorname{Pr}=0.733$.

It is seen from the Table $\mathbf{1}$ that the skin-friction coefficient increases as the viscosity parameter, the radiation parameter, thermal Grashof number or solutal Grashof number increases. But the increasing of the magnetic parameter, Schmidt number or porous parameter leads to a decrease in the skin-friction.

\section{Conclusion}

The governing equations for boundary layer flow and mass transfer of a steady viscous, incompressible elec-

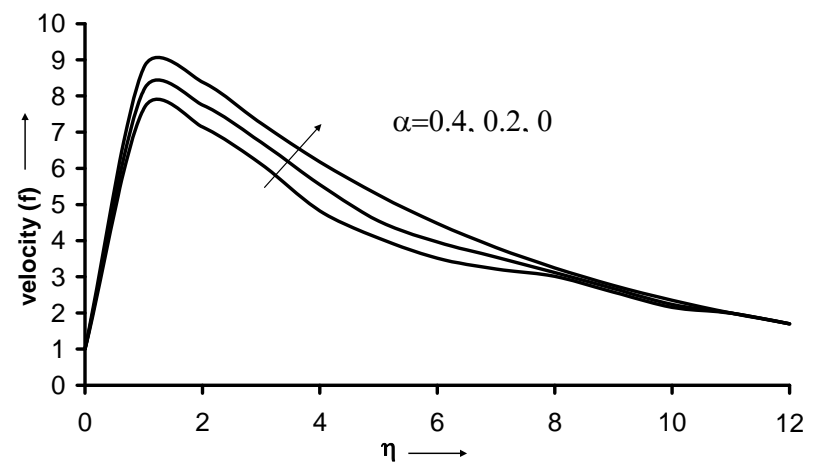

Figure 2. Velocity distribution for various values of $\alpha$ with $G r=5, R=0.2, M=2, G m=1, S c=5, k_{1}=0.5$.

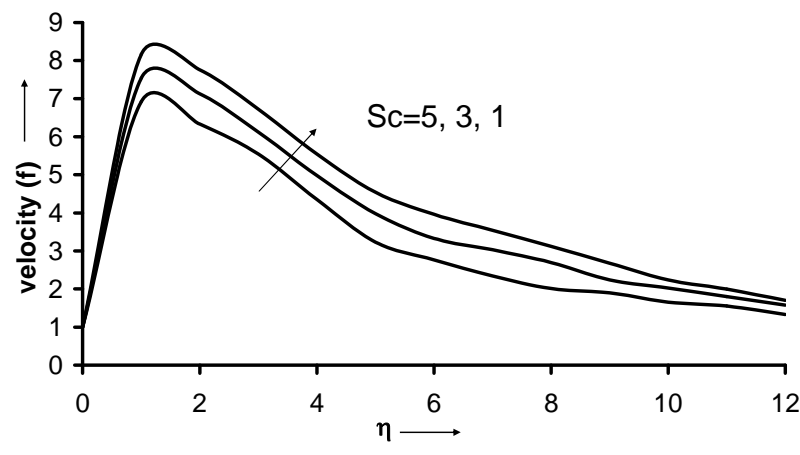

Figure 3. Velocity distribution for various values of $S c$ with $G r=5, R=0.2, M=2, G m=1, k_{1}=0.5$.

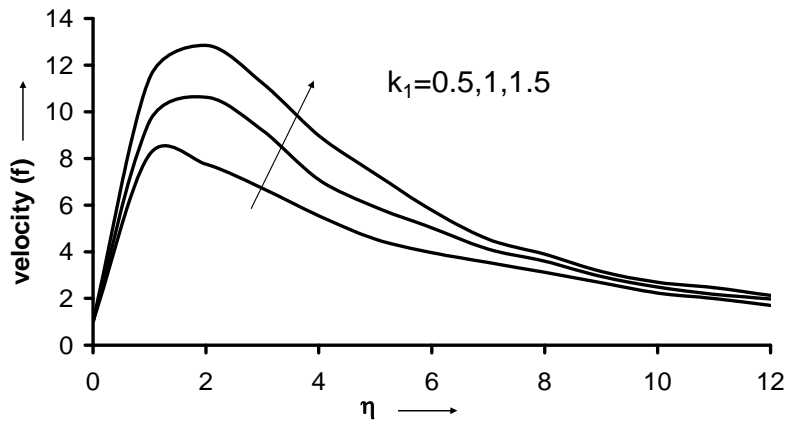

Figure 4. Velocity distribution for various values of $k_{1}$ with $G r=5, R=0.2, M=2, G m=1, S c=3$.

Table 1. Numerical values of $f^{\prime}(0)$ for different $\alpha$.

\begin{tabular}{cccccccc}
\hline$\alpha$ & $M$ & $R$ & $G r$ & $G m$ & $S c$ & $k_{1}$ & $f^{\prime}(0)$ \\
\hline 0 & 0 & 0.5 & 5 & 0.5 & 3 & 0.5 & 4.9982 \\
-0.2 & 0 & 0.5 & 5 & 0.5 & 3 & 0.5 & 2.8740 \\
-0.4 & 0 & 0.5 & 5 & 0.5 & 3 & 0.5 & -1.4110 \\
-0.2 & 1 & 0.5 & 5 & 0.5 & 3 & 0.5 & -6.4225 \\
-0.2 & 1 & 0.8 & 5 & 0.5 & 3 & 0.5 & -3.2241 \\
-0.2 & 1 & 0.5 & 7 & 0.5 & 3 & 0.5 & 1.2554 \\
-0.2 & 1 & 0.5 & 5 & 1.0 & 3 & 0.5 & 6.2445 \\
-0.2 & 1 & 0.5 & 5 & 0.5 & 5 & 0.5 & 4.9445 \\
-0.2 & 1 & 0.5 & 5 & 0.5 & 5 & 1.0 & 2.2664 \\
\hline
\end{tabular}

trically conducting fluid with variable viscosity over a continuously moving vertical porous plate in the presence of magnetic field and radiation has been investigated. It was found that when viscosity parameter and porous parameters were increased, the fluid velocity increased. However, velocity decreases as the Schmidt number decrease. In addition, it was found that the skin-friction coefficient increased due to increase in the viscosity parameter, the radiation parameter, thermal Grashof number or solutal Grashof number. But the increasing of the magnetic parameter, Schmidt number or porous parameter leads to a decrease in the skin-friction.

\section{REFERENCES}

[1] B. C. Sakiadis, "Boundary Layer Behaviour on Continuous Solid Surface: II. The Boundary Layer on a Continuous Flat Surface,” AIChE Journal, Vol. 7, No. 2, 1961, pp. 221-225. doi:10.1002/aic.690070211

[2] F. K. Tsou, F. M. Sparrow and R. J. Golldstien, "Flow and Heat Transfer in the Boundary Layer in Continuous Moving Surface,” International Journal of Heat and Mass Transfer, Vol. 10, No. 2, 1967, pp. 219-235. doi:10.1016/0017-9310(67)90100-7

[3] L. E. Erickson, L. T. Fan and V. G. Fox, "Heat and Mass Transfer on a Moving Continuous Flat Plate with Suction 
and Injection,” Industrial \& Engineering Chemistry Fundamentals, Vol. 5, No. 1, 1966, pp. 19-25. doi:10.1021/i160017a004

[4] L. J. Crane, "Flow Past a Stretching Plate," Zeitschrift für Angewandte Mathematik und Physik, Vol. 21, No. 4, 1970, pp. 645-647. doi:10.1007/BF01587695

[5] B. Rajesh Kumar, D. R. S. Raghuraman and R. Muthucumaraswamy, "Hydromagnetic Flow and Heat Transfer on a Continuously Moving Vertical Surface," Acta Mechanica, Vol. 153, No. 3-4, 2002, pp. 249-253. doi:10.1007/BF01177455

[6] P. R. Sharma and P. Mathur, "Steady Laminar Free Convection Flow of an Electrically Conducting Fluid along a Porous Hot Vertical Plate in the Presences of Heat Source/ Sink," Indian Journal of Pure and Applied Mathematics, Vol. 26, No.11, 1995, pp. 1125-1134.

[7] A. Raptis and C. V. Massalas, "Magnetohydrodynamic Flow Past a Plate by the Presence of Radiation," Heat and Mass Transfer, Vol. 34, No. 2-3, 1998, pp. 107-109. doi:10.1007/s002310050237

[8] A. J. Chamkha, "Thermal Radiation and Buoyancy Effects on Hydromagnetic Flow over an Accelerating Permeable Surface with Heat Source or Sink,” International Journal of Engineering Science, Vol. 38, No. 15, 2000, pp. 1699-1712. doi:10.1016/S0020-7225(99)00134-2

[9] A. Raptis, C. Perdikis and H. S. Takhar, "Effect of Thermal Radiation on MHD Flow," Applied Mathematics and Computation, Vol. 153, No. 15, 2004, pp. 645-649. doi:10.1016/S0096-3003(03)00657-X

[10] Md. Anwar Hossain and Md. S. Munir, "Mixed Convection Flow from a Vertical Plate with Temperature Dependent Viscosity," International Journal of Thermal Sciences, Vol. 39, No. 2, 2000, pp. 173-183.

[11] T. Fang, "Influences of Fluid Property Variation on the Boundary Layers of a Stretching Surface," Acta Mechanica, Vol. 171, No. 1-2, 2004, pp. 105-118. doi:10.1007/s00707-004-0125-y
[12] M. Anwar Hossain, K. Khanafer and K. Vafai, “The Effect of Radiation on Free Convection Flow of Fluid with Variable Viscosity from a Porous Vertical Plate,” International Journal of Thermal Sciences, Vol. 40, No. 2, 2001, pp. 115-124. doi:10.1016/S1290-0729(00)01200-X

[13] M. A. A. Mahmoud, "Variable Viscosity Effects on Hydromagnetic Boundary Layer Flow along a Continuously Moving Vertical Plate in the Presence of Radiation,” Applied Mathematical Sciences, Vol. 1, No. 17, 2007, pp. 799-814.

[14] U. N. Das, R. K. Deka and V. M. Soundalgekar, "Effect of Mass Transfer on Flow Past an Impulsively Started Vertical Plate with Constant Heat Flux and Chemical Reaction,” Forschung im Ingenieurwesen, Vol. 60, No. 10, 1994, pp. 284-287.

[15] R. Muthucumarswamy and P. Ganesan, "Diffusion and First-Order Chemical Reaction on Impulsively Started Infinte Vertical Plate with Variable Temperature," International Journal of Thermal Sciences, Vol. 41, No. 5, 2002, pp. 475-479. doi:10.1016/S1290-0729(02)01340-6

[16] K. V. Prasad, S. Abel and P. S. Datti, "Diffusion of Chemically Reactive Species of a Non-Newtonian Fluid Immersed in a Porous Medium over a Stretching Sheet," International Journal of Non-Linear Mechanics, Vol. 38, No. 5, 2003, pp. 651-657. doi:10.1016/S0020-7462(01)00122-6

[17] A. Y. Ghaly and M. A. Seddeek, "Chebyshev Finite Difference Method for the Effect of Chemical Reaction, Heat and Mass Transfer on Laminar Flow along a Semi-Infinite Horizontal Plate with Temperature Dependent Viscosity," Chaos, Solitons \& Fractals, Vol. 19, No. 1, 2004, pp. 61-70. doi:10.1016/S0960-0779(03)00069-9

[18] F. T. Akyildiz, H. Bellout and K. Vajravelu, "Diffusion of Chemically Reactive Species in a Porous Medium over a Stretching Sheet," Journal of Mathematical Analysis and Application, Vol. 320, No. 1, 2006, pp. 322-339. doi:10.1016/j.jmaa.2005.06.095 